\title{
Erratum to: From simplicity towards complexity: the Italian multidimensional approach to obesity
}

\author{
Lorenzo M. Donini - Riccardo Dalle Grave • Antonio Caretto $~$ Lucio Lucchin • \\ Nazario Melchionda $\cdot$ Enzo Nisoli • Paolo Sbraccia • Andrea Lenzi • \\ Massimo Cuzzolaro
}

Published online: 25 February 2014

(C) Springer International Publishing Switzerland 2014

\section{Erratum to: Eat Weight Disord \\ DOI 10.1007/s40519-013-0097-9}

In the original publication of the article, the name of second author was shortened incorrectly in the affiliation section. The correct name should read R. Dalle Grave.

The online version of the original article can be found under doi:10.1007/s40519-013-0097-9.

L. M. Donini $(\bowtie) \cdot$ A. Lenzi

Department of Experimental Medicine-Medical

Physiopathology, Food Science and Endocrinology Section, Sapienza University of Rome, P.le Aldo Moro, 5, 00185 Rome, Italy

e-mail: lorenzomaria.donini@uniroma1.it

L. M. Donini - N. Melchionda $\cdot$ M. Cuzzolaro

SISDCA: Italian Society for the Study of Eating Disorders,

Rome, Italy

R. Dalle Grave

Department of Eating and Weight Disorders, Villa Garda

Hospital, Garda (VR), Italy

A. Caretto $\cdot$ L. Lucchin

ADI: Italian Association of Dietetics and Clinical Nutrition,

Rome, Italy

A. Caretto

Perrino Hospital, Brindisi, Italy
L. Lucchin

Dietetic and Clinical Nutrition Unit, Regional General Hospital, Bolzano, Italy

E. Nisoli · P. Sbraccia

SIO: Italian Society of Obesity, Rome, Italy

E. Nisoli

Department of Medical Biotechnology and Translational

Medicine, University of Milan, Milan, Italy

P. Sbraccia

Department of Systems Medicine, University of Rome Tor Vergata, Rome, Italy

A. Lenzi

University College of Italian Endocrinologists, Rome, Italy

A. Lenzi

SIE: Italian Society of Endocrinology, Rome, Italy 